\title{
On the Demonstration and Evaluation of Service- Based Slices in 5G Test Network using NFV
}

\author{
Muhammad Arif \\ Centre for Wireless Communications \\ University of Oulu, Finland \\ muhammad.arif@oulu.fi
}

Ari Pouttu
Centre for Wireless Communications
University of Oulu, Finland ari.pouttu@oulu.fi

\author{
Olli Liinamaa \\ Centre for Wireless Communications \\ University of Oulu, Finland \\ olli.liinamaa@oulu.fi \\ Mika Ylianttila \\ Centre for Wireless Communications \\ University of Oulu, Finland \\ mika.ylianttila@oulu.fi
}

\author{
Ijaz Ahmad \\ Centre for Wireless Communications \\ University of Oulu, Finland \\ ijaz.ahmad@oulu.fi
}

\begin{abstract}
This paper demonstrates the realization of different network services, 5G Test Network (5GTN) as a mobile network operator at the University of Oulu provides based on an open access network availability for research and innovation. A network platform with use case and service dedicated slicing is designed on the fundamental principles of ETSI compliant NFV Management and Network Orchestration (MANO) framework. The slicing is done primarily on the Core Networks (CN) with evolved packet core and ip multimedia subsystem functionalities in different slices. The virtual network function instances are instantiated in OpenStack and VMWare cloud environments using an NFV Orchestrator. The virtual instances and the required resources can be instantiated, terminated and scaled in a slice according to the requirements of the end user and nature of the use case. Focusing on different service demands, the paper demonstrates three test cases utilizing different slices from the components in the $\mathrm{CN}$ for each case. It also provides an overview on radio spectrum sharing possibility between two operators on a specified service level agreement with each operator having own CN infrastructure.
\end{abstract}

\section{Keywords-5GTN, Slicing, NFV, MANO, Orchestrator}

\section{INTRODUCTION}

Over the years, the expectations towards a Mobile Network Operator (MNO) in context of availability and diversity of offered services has changed significantly [1],[2]. As an outcome of the expectations from future generations of cellular networks to host variety of verticals, the network infrastructure has constantly been evolving and is getting accustomed to the expected growth of end user needs [1]. In context of current cellular network infrastructure and the first $5 \mathrm{G}$ commercialization, MNOs together with the inclusion of all the requisite entities should modify the current infrastructure to come up with a platform which would be capable to ensure realization of $5 \mathrm{G}$ use cases and service delivery to the end users.

Network Functions Virtualization (NFV), Network Slicing (NS) and Cloud Computing is a requisite technological addition in cellular networks to tackle the diversity in requested services by individual subscribers. For instance, autonomous vehicles in contrast to media content delivery is delay sensitive and yet requires reasonably less bandwidth [3]. Moreover, MNOs have been facing challenges to manage network resources efficiently since several decades. The legacy cellular networks primarily comprised of proprietary and bulky hardware boxes which were inefficient in terms of Capital Expenditure (CAPEX) and Operational Expenditure (OPEX) [4]-[6]. The maintenance and troubleshooting of such a hardware was challenging for the operators. For instance, in an event of a network crash due to a faulty hardware, there is a large time lapse expected before the hardware becomes functional which indeed compromises the Quality of Service (QoS) delivered to the customers. With the evolution of cellular networks, Network Softwarisation and Slicing started emerging as an alternate to hardware centric networks to overcome these problems [4]-[6].

$\mathrm{NFV}$, the standard IT technique of virtualizing network functions, by the abstraction of network functions from the physical resources of a hardware makes the software instances independent of the underlying hardware [7]. Such an abstraction is achieved by using a hypervisor layer between the hardware and the operating system running the network functions as software instances [3]. The virtual resources can then be efficiently distributed among Virtual Network Functions (VNFs) which helps MNOs to tackle load balancing and resource management issues in real networks [4]. Network slicing on the other hand ensures the allocation of optimized and service specific resources based on the demand of end users [7].

This paper provides an analysis of 5GTN on an infrastructural level to showcase service-based NS enabled by leveraging NFV principles. This paper from here on is organized as follows: Section II advocates on the fact that NFV is a key enabler to make NS possible. Section III gives an analysis of 5GTN from an architectural to different service availability aspects. Section IV explains the experimental setup within 5GTN for testing User Equipments (UEs) with different service demands. Section V documents all the acquired results and evaluate them. Section VI provides a discussion on future research targets on the roadmap with references to the remarkable work recently carried out on NS even before its standardization and it also highlights few testbed platforms focusing on slicing. Finally, Section VII will provide with concluding remarks in a nutshell. 


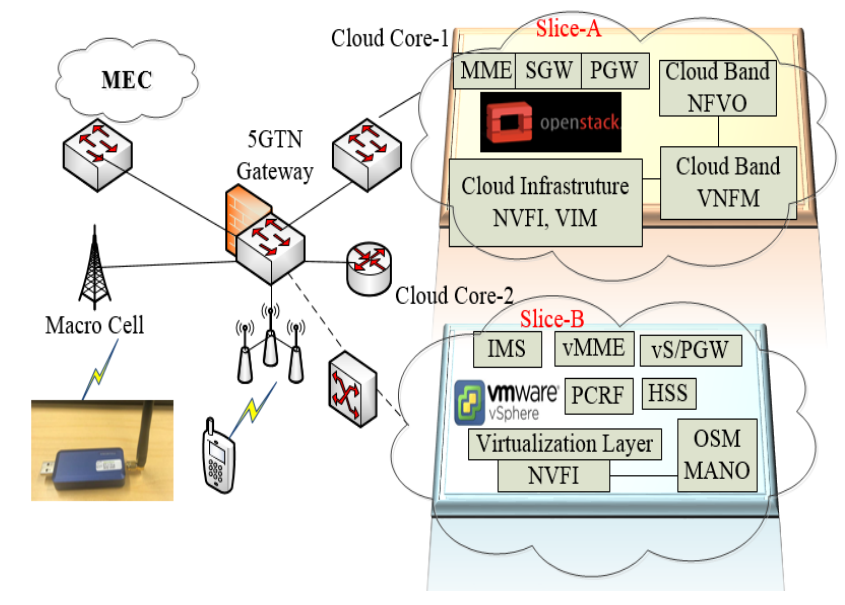

Fig. 1. Service-based network slices in 5GTN.

\section{NFV AS A KEY ENABLER FOR NETWORK SLICING}

This section provides an overview on NFV and NS. The idea is to advocate the claim that NFV is an enabler technology for NS in mobile networks.

\section{A. Network Functions Virtualization}

NFV is the abstraction of network functions from the underlying hardware by any virtualizing technology and deploying them as software instances anywhere in a network at any time instant. Such a decoupling eventually results in cost efficiency due to the NFV MANO taking care of the efficient resource management. Network Functionalities are instantiated as VNFs in Virtual Machines (VMs) on demand and then later terminated, scaled and healed depending on the network conditions and end user requirements. These VMs are deployed in Virtual Infrastructure Manager (VIM) which are basically cloud platforms like OpenStack, VMWare, Amazon etc. Having the network, compute and storage resources, the hardware underlying these clouds with a virtualization technology in between is termed as Network Function Virtualization Infrastructure (NFVI). Because of NFV, the functional entities of a mobile core network, for instance Mobility Management Entity (MME), Serving/Packet Gateway (S/PGW), Policy Control and Charging Function (PCRF), Home Subscriber Server (HSS) etc, can be deployed as virtual instances which as a result makes the network flexible, dynamic and ensures better quality of service. [1],[4],[6],[7],[9]

\section{B. Network Slicing}

Network Slicing is a methodology of allocating requisite resources to individual users based on their service requirements by the instantiation of virtual instances from Radio Access Network (RAN) all the way to the CN [7],[9]. According to 3 GPP and ITU-T, NS enables creation of logically isolated network partitions primarily consisting of virtual instances which can be optimized and tailored for diverse service demands [7],[10],[11]. In context of dedicated service delivery to end users in a mobile network, NS scenario is well explained in [9] which distributes users into hierarchies based on different use cases. In order to realize NS in operator networks, Wireless Network Virtualization (WNV), Software Defined Networking SDN) and NFV are the key enablers [7],[9]. WNV is the sharing

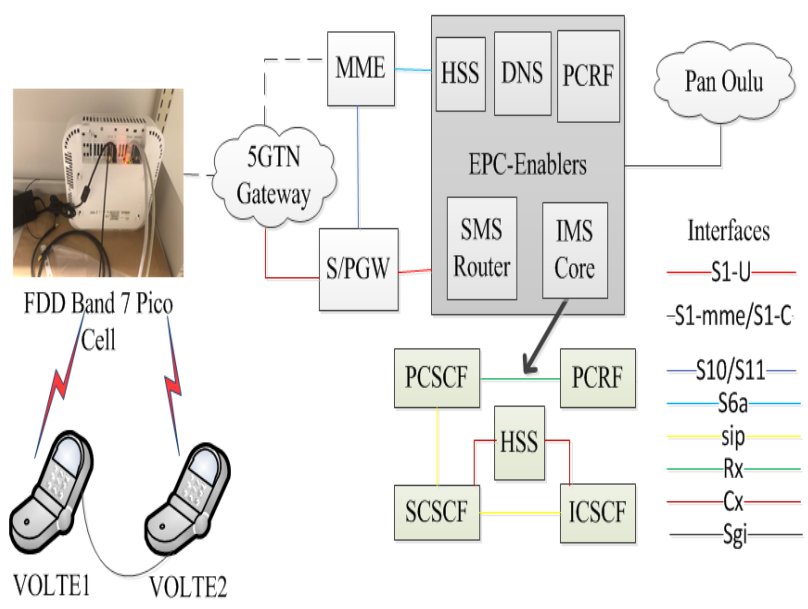

Fig. 2. VoLTE call over IMS in 5GTN.

of radio spectrum and infrastructure resources by effective slicing to efficiently distribute them among users demanding various services with different QoS levels and SDN is the technology enabling the complete separation of Control and Data Plane [7],[9],[23].

\section{5G TEST NETWORK}

The 5G Test Network (5GTN) is an open testbed for next generation mobile networks and it provides innovation possibilities to service developers and network operators [12]. 5 GTN at the University of Oulu, comprises RAN operating on licensed LTE and 5G bands [12]. The $\mathrm{CN}$ resources includes Virtualized Evolved Packet Core (EPC) and IP Multimedia Subsystem (IMS) components in different cloud runtimes in a multi-slice environment as shown in Figure 1. To provide low latency services, 5GTN also contains Mobile Edge Computing at the edge of the network [12]. Figure 1 gives an overview on the different slices which are tailored for specific services with a common gateway. The network slices shown in Figure 1 is specified to the $\mathrm{CN}$ slicing only and currently there is no slicing in the RAN. Although this multi-core model is not the same as intended slicing in 5G core [16], this slicing model however enables resource tailoring according to service requirements and brings valuable research input targeting $5 \mathrm{G}$ core and more optimized slicing functionality. Due to such service provisioning flexibility, an MNO can deploy instances of $\mathrm{CN}$ all at once to serve UEs categorized based on their service requirements [7]. The EPC and IMS components i.e. MME, S/PGW, HSS, Serving Call Session Function (SCSCF), Proxy Call Session Function (PCSCF) and Interrogating Call Session Function (ICSCF) are deployed in cloud environments such as OpenStack and VMware. The network imitates multi-core network model which focuses on sharing the RAN and spectrum in which operators maintains separate EPC instances and base stations are connected using separate S1 interfaces [7][8]. Through a single gateway, each base station is connected to a slice consisting of virtual instances providing a set of services. 'Slice-A' as depicted in Figure 1 provides Enhanced Mobile Broadband (eMBBs) and high-speed LTE data services, ensuring high speed data connectivity with high bandwidth which is ideal for media content delivery. Along with that, this slice also enables Internet Of Things (IoT) applications due to the Narrow 


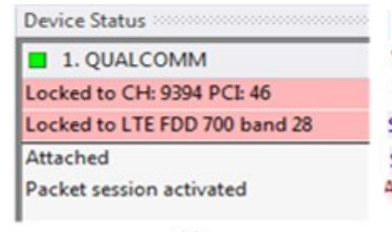

(a)

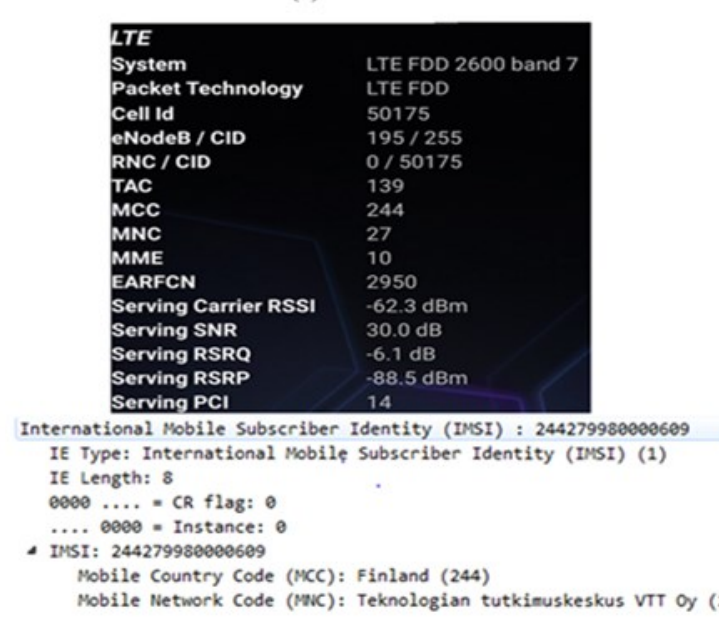

INVITE sip:+490001@ims.mnce01. mcce01.3gppnetwork.org; user=phone SIP/2.0 Via: SIP/2.0/TCP $19 \overline{2} .168 .3 .152: 5060$ SIP/2.0 100 Trying $\mathrm{SIP} / 2.0180$ Ringing SIP/2.0 200 OK ACK sip: $192.168 .3 .151: 5060 \mathrm{SIP} / 2.0$

(b)

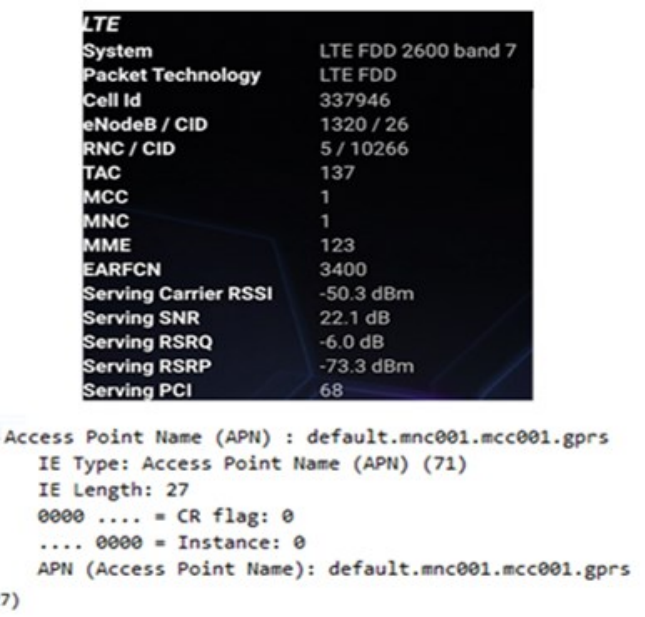

(c)

Fig. 3. a) Status of the Qualcomm NB-IoT Dongle b) Wireshark logs of the SIP call flow c) Network switching on a specified SLA.

Band IoT (NB-IoT) capability in the RAN and the CN. The RAN for this slice operates on LTE band 7, Band 28 and band 42. The cells on this slice broadcasts on ' 24427 ' Public Land Line Mobile Network (PLMN). 'Slice-B' on the other hand, with the support of policy control and IMS is dedicated for critical communications and Voice Over LTE (VoLTE). Additionally it provides basic data services. The RAN for this slice comprises of LTE band7 Pico cells. The cells in this slice are broadcasting on test PLMN values i.e. '00101'. Each slice has its own virtual core network functions realized by NFV technology as VNFs in different clouds. The efficient resource management is ensured by the dynamic allocation of resources by NFV MANO. The VNFs are instantiated, terminated, healed and scaled according to the service level demands to efficiently utilize the underlying hardware resources. The credentials of the mobile subscribers are provisioned in the HSS of each slice and can switch the network to get different services by the change of Access Point Name (APN) on the end device. The slicing of the wireless network on the RAN is a work in progress and is out of the scope of this paper.

\section{THE EXPERIMENTAL SETUP}

\section{A. NB-IoT module connectivity and tests over Slice-A}

In order to exploit IoT services for realizing different use cases, 'slice-A' in 5GTN provides NB-IoT support both on the RAN and the CN. As depicted in Figure 1, a Qualcomm's NBIoT module with LTE band 28 support is connected to the network through a base station having an S1 interface to the mme of 'Slice-A' which is been instantiated in Cloud Core-1. '5gtnouluiot' is the 'APN' used for NB-IoT connectivity in the Qualcomm device. Additionally, using this slice, an end device can have basic LTE data services as well. However, the APN in this case needs to be changed to ' 5 gtnoulu'.

\section{B. VoLTE service using Slice- $B$}

VoLTE is a support for voice communication in LTE networks which requires Adaptive Multi Rate Wide Band (AMR-WN) codec, PCRF for QoS and IMS [13],[15]. VoLTE traffic primarily comprises Session Initiation Protocol (SIP) which is a signaling protocol used to create/modify/terminate a session between two users [13]. To showcase voice call functionality in 5GTN using IMS core functionalities in the 'Slice-B', two UEs are connected via a base station with an S1 interface to the $\mathrm{CN}$ of 'Slice-B' in Cloud Core-2. Figure 2 demonstrates the experimental setup in which a subscriber VoLTE1 performs a VoLTE call to subscriber VoLTE2 utilizing the IMS call session functions. 'IMS' is the APN used in the UEs to perform a VoLTE call based on the pre-defined enforced policies from the PCRF.

\section{Network re-selection by change in the APN}

Based on the subscriber provisioning in both $\mathrm{CN}$, for acquiring basic LTE data services, a UE can be connected to $\mathrm{CN}$ in different slices depending on the use cases by a mere change of APN. It is important to note here that the base station commissioning is done keeping in view the mme IP address, Tracking Area Code (TAC) and PLMN values of each CN in Slice-A and B which have already been instantiated in Cloud Core- 1 and 2 respectively.

\section{EVALUATION AND RESUlts}

Utilizing the experimental setup, various experiments are conducted, showcasing the possibilities of different service delivery in 5GTN. The results are hereby analyzed and evaluated as follows: 


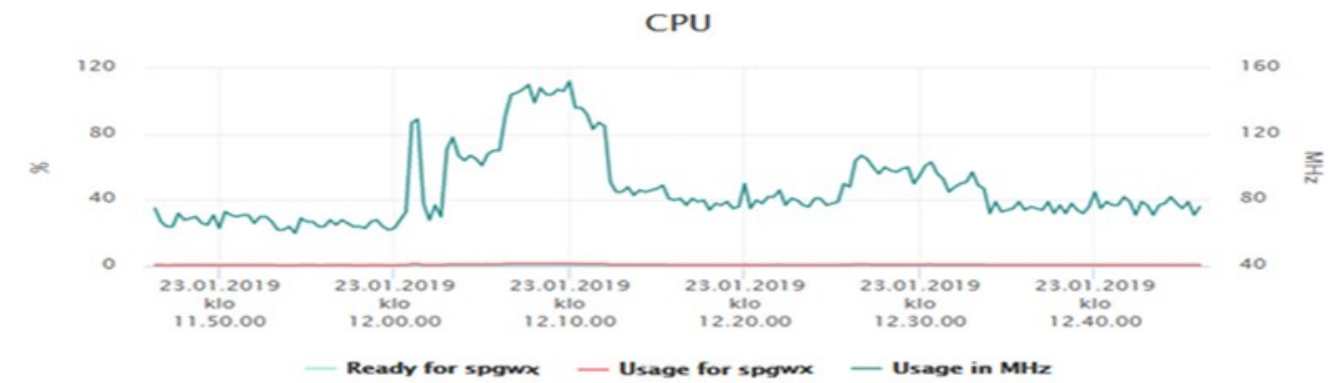

(a)

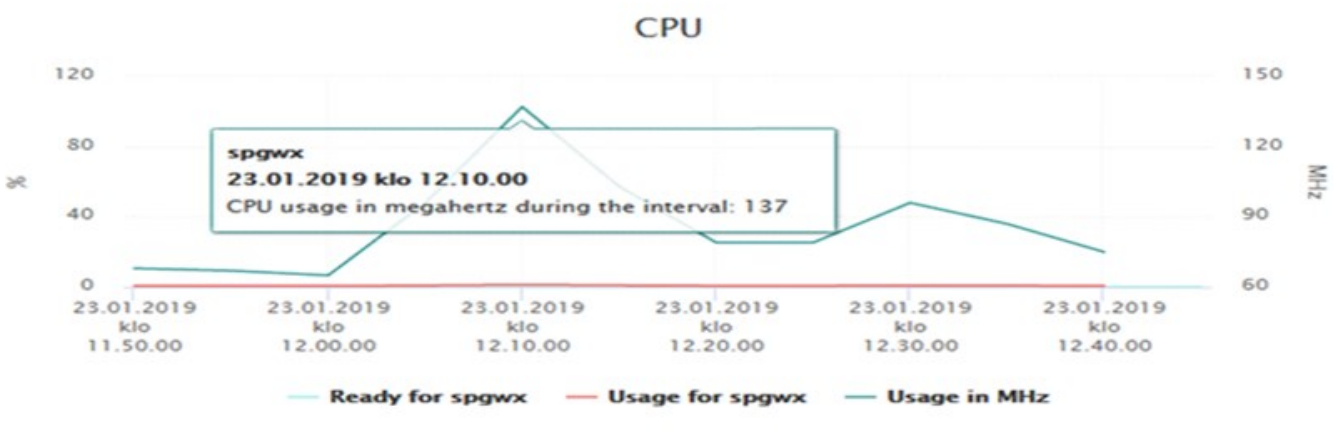

(b)

Fig. 4. a) Real-time CPU utilization on the S/PGW b) CPU utilization for custom period during service delivery.

\section{A. NB-IoT connectivity test}

The experimental result in Figure 3 (a) is carried out using Nemo Outdoor measurement and analysis tool powered by Keysight Technologies [18]. The connection status of the NBIoT module is shown with channel, Physical Cell Identity (PCI) and LTE band level information. Along with the connectivity, it depicts the creation of a packet session validating the support of NB-IoT services in 5GTN in a dedicated service slice.

\section{B. VoLTE call}

Based on the experimental setup in Figure 2, a VoLTE call is triggered between two UEs. Both the UE registers initially to the network with the creation of default bearer using 'Slice-B'. The UEs then gets the IMS registration by a SIP registration process which involves PCSCF, ICSCF and SCSCF nodes. To validate a successful VoLTE call between two UEs using Slice$\mathrm{B}$, a Wireshark log is taken from the virtual interfaces from the VNF containing the IMS as shown in Figure 3 (b). Whereas the IMS nodes containing the call session functions are presented in the experimental setup in Figure 2.

VoLTE1 initiates a voice call by sending the 'SIP invite' message containing the session description function offered with media and bandwidth information. The 'SIP invite' message is then acknowledged by VoLTE2 after sending a 100 Trying message. After the acknowledgment dedicated bearers are created at both VoLTE1 and VoLTE2 by enforced policies of the PCRF. Once the media stream is active by the resource reservation confirmation, the VoLTE2 starts to ring and then it replies with 'SIP 180 Ringing' message and response as shown in Figure 3 (b). Once VoLTE2 receives the call, we get a 'SIP 200 Ok' message. Finally, 'SIP ACK' message shows that call has been established between VoLTE1 and VoLTE2 over a dedicated bearer. [19]

\section{Switching End user for internet access}

'NemoHandy', an indoor software analytical tool powered by KeySight Technologies [18], is used in the UE for logging the network parameters. As depicted in Figure 3 (c), the UE is switched from 'Slice-A' to 'Slice-B' by the change of APN. Figure 3 (c) on the left side represents a UE connected via band 7 base station to 'Slice-A' using ' 5 gtnoulu'APN and on the right side shows the UE connected via band 7 base station to 'Slice-B' using 'default' APN. The TAC and PLMN values are ' 139 ' and '24427' respectively when the UE connects to 'SliceA' and with the change of APN to 'default' when the UE gets connected to Slice-B, the TAC and PLMN values are '137' and '00101' respectively. Additionally, Wireshark logs in Figure 3 (c) from the respective MME node, gives International Mobile Subscriber Identity (IMSI), PLMN and the used APN. The switch of the UE can additionally be validated by the difference in MME ID and PCI values.

To showcase the CPU consumption for service delivered using Slice-B and Cloud Core-2, real time and custom performance is provided in Figure 4 . Figure 4 (a) presents, the $\mathrm{CPU}$ utilization (in percentage and $\mathrm{MHz}$ ) at the $\mathrm{S} / \mathrm{PGW}$ for two UEs constantly streaming a live video feed during 12:00:00$12: 10: 00$ and a voice call during 12:26:00-12:33:00 on 23.1.2019. Whereas Figure 4 (b) gives a more visible view of the CPU utilization during the service delivery time intervals. It is evident from the results that the CPU utilization started increasing as an incline ramp, once the UEs started streaming videos and reached to its maximum at 12:10:00. At this point, it started decreasing as the video streaming was paused on both UEs. Additionally, in Figure 4 (b), the CPU utilization during the VoLTE call between two UEs can be seen between 12:26:00-12:33:00. The smaller ramp on the right side of Figure 


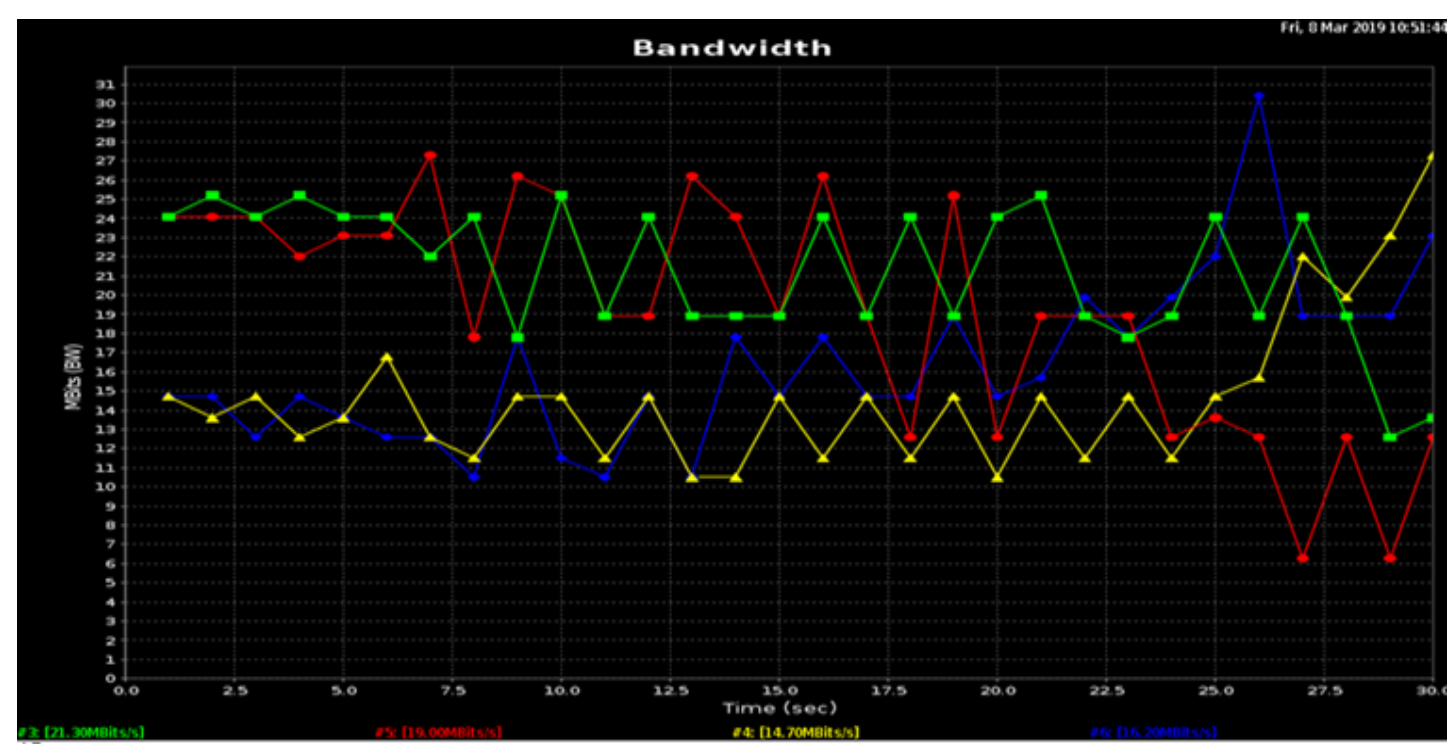

Fig. 5. Throughput measurements in Slice B (UE as server and S/PGW as client).

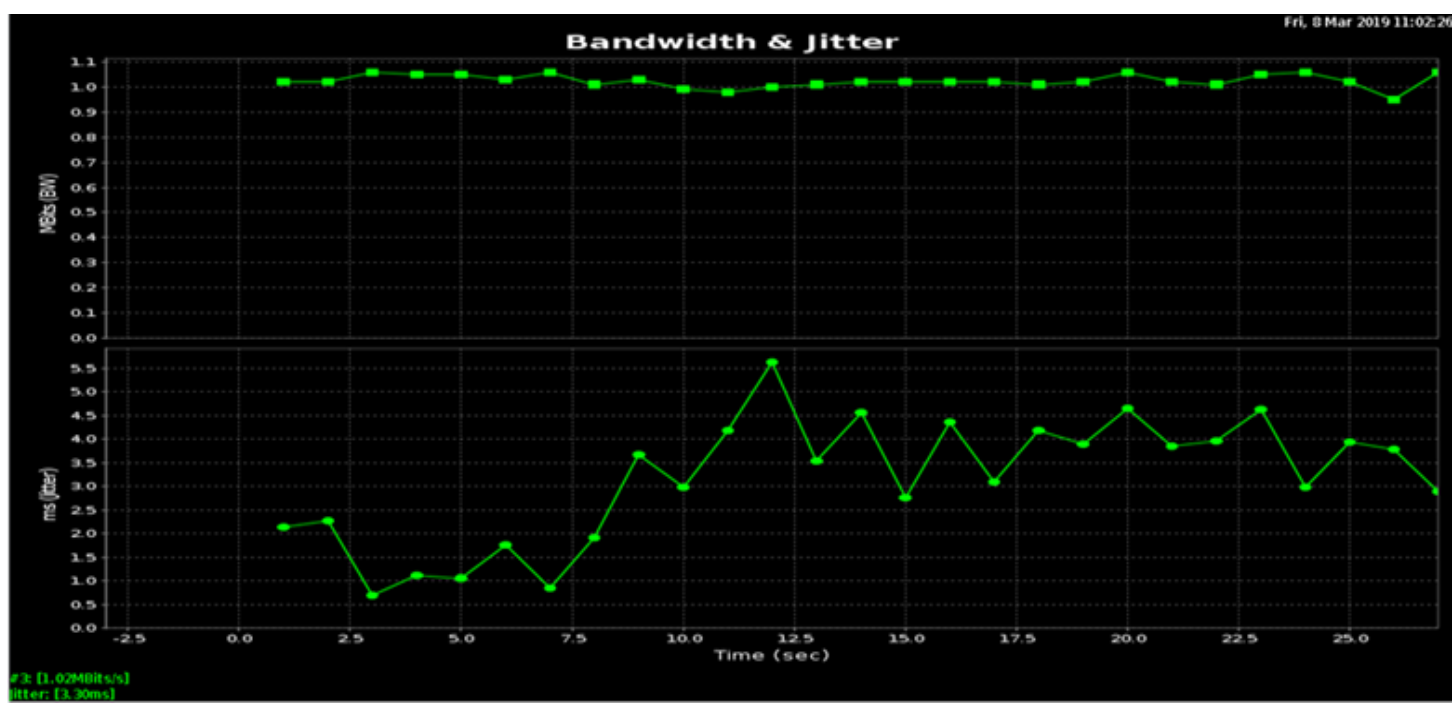

Fig. 6. Variation in network delay within each RTT response time.

4 (b) is because of the placed VoLTE call. It started with an increase in CPU percentage than normal and reached to its maximum at 12:33:00 and started declining. It is evident from the analysis that the CPU utilization on the $\mathrm{S} / \mathrm{PGW}$ is more than double for media delivery in contrast to voice call.

A performance analysis is also carried out in terms of throughput and latency within the internal network. Jperf, the graphical version of Iperf tool is utilized to measure the maximum achievable bandwidth. An iperf server is created at the S/PGW of the Slice B and an iperf client at the attached UE. Figure 5 illustrates the achieved throughput of $71.20 \mathrm{MBits} / \mathrm{s}$ on the downlink by running 4 parallel streams for thirty seconds with one second intervals. It is the sum of throughput of all the four streams. It is important to note here that the channel bandwidth on the base station is $10 \mathrm{MHz}$ with $2680 \mathrm{MHz}$ as the center frequency. Figure 6 depicts the jitter value which is the variation in the round trip time (RTT) delay from the UE to the
$\mathrm{S} / \mathrm{PGW}$. This result is acquired by creating an iperf server at the SPGW listening to UDP streams on port 5001 and an iperf client on the attached UE. The test is run for thirty seconds with a single stream. We can observe that the Jitter value is $3.20 \mathrm{~ms}$ which corresponds to the average variation or inconsistency in the RTT delay. In order to observe the RTT values, a ping test is also carried out at the UE by pinging the IP address of the $\mathrm{S} / \mathrm{PGW}$ on the data plane. We observed that the average RTT value was $31 \mathrm{~ms}$.

\section{DISCUSSION AND LESSONS LEARNED}

To study and experiment verticals related to $5 \mathrm{G}$, different testbeds were deployed across the globe. The Eindhoven $5 \mathrm{G}$ Brainport platform in the TU/e campus [20], the testbed at the Budapest University of Technology and Economics [21] and an open source $5 \mathrm{G}$ testbed for slice evaluation [22] are some of the $5 \mathrm{G}$ platforms being utilized for research on $5 \mathrm{G}$ use cases. 
Studying the ongoing research carried by the utilization of these testbeds have helped us understanding the significance of network slicing in delivering $5 \mathrm{G}$ use cases. Nevertheless, further improvements in our testbed are needed both on an infrastructural and design level to address end to end slicing. In light of the study on the state of the art on NS in [7], to ensure an end to end NS, the RAN slicing is requisite. The NS in 5GTN is currently limited to the $\mathrm{CN}$ which can further be enhanced towards dedicated $\mathrm{CN}$ slicing by introducing slicing in the RAN. Along with that, we are also targeting to achieve an end to end NS model in line with 3 GPP release 13 DECOR and eDECOR concepts as described in [14]. The research work in [14] shows the use of eDECOR to enforce NS at the CN level by hard coding a slice ID into UE or encoded through PLMN which in turn allows the base stations to assign UEs to requested slices [7],[14],[17]. The CPU performance, throughput and delay benchmarks have helped us evaluating the platform readiness for $5 \mathrm{G}$ use cases. Apart from performance optimization, our target in the future is to make an end to end network slice capability in 5GTN where instead of static commissioning of individual base stations, UEs should be dynamically linked up to the associated slice by the RAN. Moreover, our target is to further study and implement, the slice discovery and selection functionality introduced in 3GPP release 15 [16] to ensure slices to appropriate UEs

\section{CONCLUSION}

The experimental work performed in 5GTN through this paper showcased how NFV serves as a key enabler for creating service based-slices in an operator's network. Keeping in view the diversity of use cases as $5 \mathrm{G}$ is about to roll out, this paper performs tests on some of them by making use of network slices in the CN. Network support for devices running IoT applications is tested by connecting a commercial NB-IoT device over LTE band 28. For better voice call quality, VoLTE call is demonstrated using a dedicated network slice. Finally, the static switcing of UEs to different $\mathrm{CN}$ slice is showcased. CPU bendmarking from one of the network slice in a real time and the time during which services are delivered is presented to differentiate between the resource usage by different services delivered to the end user. Throughput and delay are also measured within one of the slice to showcase the network performance with respect to performance metrics critical to $5 \mathrm{G}$.

\section{ACKNOWLEDGMENT}

This work has been performed utilizing the 5GTN infrastructure and resources at Centre for Wireless Communications, University of Oulu. The integration work is an outcome of research activities in the CORNET project. This research has also been financially supported by Academy of Finland 6Genesis Flagship (grant 318927).

\section{REFERENCES}

[1] I. Afolabi, M. Bagaa, T. Taleb and H. Flinck, "End-to-end network slicing enabled through network function virtualization," in CSCN IEEE Conf., 2017, pp. 30-35.

[2] W. Guan, et al., "A Service-Oriented Deployment Policy of End-to-End Network Slicing Based on Complex Network Theory," IEEE Access mag., vol. 6, pp. 19691-19701, 2018.

[3] T. Taleb, et al., "PERMIT: Network Slicing for Personalized 5G Mobile Telecomms," IEEE Comms Mag., vol. 55, no. 5, pp. 88-93, 2017.

[4] W. Lee and H. Kim, "Deployment Scenario and Architecture of MANO for NFV Network Services," in ICISS, 2016, pp. 1-3.

[5] D. Lake et al., "Virtualising and orchestrating a 5G evolved packet core network," in IEEE Conf. on NetSoft, 2017, pp. 1-5.

[6] H. Hawilo, A. Shami, M. Mirahmadi and R. Asal, "NFV: state of the art, challenges, and implementation in next generation mobile networks (vEPC)," IEEE Netw., vol. 28, no. 6, pp. 18-26, 2014.

[7] I. Afolabi, et al., "Network Slicing and Softwarization: A Survey on Principles, Enabling Technologies, and Solutions," IEEE Comms Surveys \& Tutorials, vol. 20, no. 3, pp. 2429-2453, 2018.

[8] Network Sharing; Architecture and Functional Description, Release 12, 3GPP Standard TS 23.251, Mar. 2015.

[9] M. Richart, J. Baliosian, J. Serrat and J. Gorricho, "Resource Slicing in Virtual Wireless Networks: A Survey," IEEE Trans on Netw. and Service Mgmt, vol. 13, no. 3, pp. 462-476, 2016.

[10] "Framework of network virtualization for future networks, next generation network-Future networks," Int. Telecommun. Union, Geneva, Switzerland, ITU-T Recommendation Y.3011, 2012.

[11] "Study on architecture for next generation system, release 14," 3GPP, Sophia Antipolis, France, Rep. TR 23.799, 2016

[12] 5G Test Network: "Explore 5G features and performance in a controlled, real environment." [Online]: Available: https://5gtn.fi/

[13] A. E. Ko, et al., "SIP amplification attack analysis and detection in VoLTE service network," in ICOIN, 2016, pp. 334-336.

[14] A. Ksentini and N. Nikaein, "Toward Enforcing Network Slicing on RAN: Flexibility and Resources Abstraction," IEEE Comms Mag., vol. 55 , no. 6, pp. 102-108, 2017.

[15] J. Hyun, et al., "A VoLTE traffic classification method in LTE network," Asia-Pacific Netw. Operations and Mgmt. Symposium, 2014, pp. 1-6.

[16] System Architecture for the 5G System, Rel. 15, 3GPP Standard TS23.501, 2017.

[17] "Enhancement of dedicated core networks selection mechanism, rel.14," 3GPP, Sophia Antipolis, France, Rep. 3GPP TR 23.711, 2016.

[18] Nemo Wireless Network Solutions. [Online] Available: https://www.keysight.com/en/pc-2767981/nemo-wireless-network-solut ions? $\mathrm{nid}=-32104.0 .00 \& \mathrm{cc}=\mathrm{FI} \& \mathrm{lc}=$ fin

[19] V. Gurbani, Ed., et al., "Session Initiation Protocol (SIP) Overload Control", RFC 7339, DOI 10.17487/RFC7339, 2014,

[20] I. T. Monroy et al., "Testing Facilities for End-to-End Test of Vertical Applications Enabled by 5G Networks: Eindhoven 5G Brainport Testbed," in ICTON, 2018, pp. 1-5.

[21] L. Nagy and Z. Szalay, "5G Wireless Test Network Design at BME," Intern. Symposium on CSNDSP,2018, pp. 1-6.

[22] A. Shorov, "5G Testbed Development for Network Slicing Evaluation," in EIConRus, 2019, pp. 39-44.

[23] I. Ahmad, S. Namal, M. Ylianttila and A. Gurtov, "Towards software defined cognitive networking," in Intern. Conf. on NTMS, 2015, pp. 1-5. 